\title{
Evaluating the usefulness of the ICT tuberculosis test kit for the diagnosis of tuberculosis
}

\author{
Chulhun Ludgerus Chang, Eun Yup Lee, Han Chul Son, Soon Kew Park
}

\begin{abstract}
Background-Early diagnosis of tuberculosis is crucial, especially in Korea, where tuberculosis is endemic.

Aims-To evaluate the validity of the ICT tuberculosis test (ICT) in early diagnosis of tuberculosis.

Methods-Sixty eight patients with tuberculosis were tested; 37 had no history of previous tuberculosis (patient group 1), and 31 had reactivated tuberculosis (patient group 2). The control groups comprised 77 subjects: 25 healthy adults, 35 hospital workers, and 17 inpatients with non-tuberculous respiratory diseases.

Results-The diagnostic sensitivities of ICT were $73 \%$ in patient group 1 and $87.1 \%$ in patient group 2 . In two patients with extrapulmonary tuberculosis, both tested positive using ICT. The specificities of ICT were $88 \%, 94 \%$, and $94 \%$ in healthy adults, hospital workers, and nontuberculous patients, respectively.

Conclusions-ICT is a useful tool for the diagnosis of tuberculosis.

(f Clin Pathol 2000;53:715-717)
\end{abstract}

Keywords: serological diagnosis of tuberculosis; ICT tuberculosis test

The diagnosis of tuberculosis has traditionally depended on the chest $x$ ray, the acid fast bacilli (AFB) stain, and AFB culture of sputum. However, these conventional methods are unsatisfactory because the AFB stain is insufficiently sensitive, and the AFB culture takes more than three weeks to produce results. More sensitive and specific tests, such as the polymerase chain reaction (PCR) and transcription mediated amplification (TMA), are inappropriate for routine laboratory testing to diagnose tuberculosis because they require technologies and equipment that are expensive or difficult to operate.

The recently introduced antibody detection methods, which use antigens such as lipoarabinomannan (LAM), ${ }^{1} \mathrm{~A} 60,{ }^{2}$ tuberculous glycolipids (TBGLs), ${ }^{3}$ and the $38 \mathrm{kDa}$ antigen, show relatively high sensitivity in the diagnosis of tuberculosis. Of those antigens, the $38 \mathrm{kDa}$ antigen is a lipoprotein found only in the Mycobacterium tuberculosis complex, and the enzyme linked immunosorbant assay (ELISA) using a monoclonal antibody to that antigen has high sensitivity and specificity in diagnosing tuberculosis. ${ }^{45}$ It is uncertain, however, whether the test is suitable for screening or diagnosing tuberculosis in Korea because the extent of the antibody response to some mycobacterial antigens shows geographical variation, ${ }^{6}$ and because the rate of BCG vaccination and the incidence of tuberculosis vary from country to country. Our study attempted to evaluate the validity of the serological antibody test for diagnosing tuberculosis. It used the recently introduced ICT tuberculosis test (ICT; ICT Diagnostics, Sydney, Australia): an antibody test kit that detects antibodies to five antigenic determinants that are fixed to a nitrocellulose membrane.

\section{Materials and methods}

The 68 patients with tuberculosis were either outpatients or inpatients at Pusan National University Hospital (PNUH), Pusan Medical Center (PMC), St Benedict Hospital, or Koo's Chest Clinic. The diagnoses of pulmonary tuberculosis were made as follows. Fifty four were culture positive for $M$ tuberculosis. The identification of $M$ tuberculosis was performed by means of the Accuprobe culture confirmation kit (Gen-Probe, San Diego, California, USA). In 12 patients, including five reactivating cases, AFB stains or cultures were negative, but typical chest $x$ ray or computed tomography (CT) findings of consolidations, cavitary lesions, or pleural effusions were shown to improve after antituberculous treatment. Blood tests in the male 25 year old patient with tuberculous meningitis showed 870 white blood cells $/ \mu \mathrm{l}$ (lymphocytes, $56 \%$ ), $280 \mathrm{mg} /$ litre of glucose, and $6300 \mathrm{mg} /$ litre of protein, and he was confirmed to have tuberculosis by PCR. ${ }^{7}$ For the 40 year old female patient with tuberculous lymphadenopathy, AFB stain and culture were not performed, and diagnosis relied upon typical histopathological findings. Group 1 comprised 37 patients with no previous history of tuberculosis, of whom 33 had pulmonary tuberculosis, two had pulmonary tuberculosis combined with intestinal tuberculosis, one had tuberculous lymphadenopathy, and one had tuberculous meningitis. AFB stain results were nine negative and 27 positive. For the patient with tuberculous lymphadenopathy, AFB stain was not done. Group 2 comprised 31 patients with a previous history of tuberculosis who had not had successful treatment or had a relapse after a complete cure; 30 had pulmonary tuberculosis, and one had pulmonary tuberculosis combined with tuberculous spondylitis. AFB stain results were five negative and 26 positive. The blood was drawn from the patients just after diagnosis or within one month after the initiation of antituberculosis treatment. All the specimens were taken between September and December of 1998.

The healthy adults were 25 people who visited the health promotion centre of PNUH. 
Table 1 Age and sex distribution, and ICT tuberculosis test results of the tested groups

\begin{tabular}{|c|c|c|c|c|c|}
\hline Tested groups & $\begin{array}{l}\text { No. of cases } \\
(M: F)\end{array}$ & $\begin{array}{l}\text { Age range } \\
\text { (mean) }\end{array}$ & $\begin{array}{l}\text { No. positive } \\
(\%)\end{array}$ & $\begin{array}{l}\text { Sensitivity } \\
(\%)\end{array}$ & $\begin{array}{l}\text { Specificity } \\
(\%)\end{array}$ \\
\hline Healthy adults & $25(7: 18)$ & $22-63(48.4)$ & 3 & & 88 \\
\hline Hospital workers & $35(4: 31)$ & $25-55(34.8)$ & $2(6)$ & & 94 \\
\hline Patients with NTRD & $17(14: 3)$ & $37-83(60.8)$ & $1(6)$ & & 94 \\
\hline Patient group 1 & $37(18: 19)$ & $19-87(38.4)$ & $27(73)$ & 73 & \\
\hline Smear $(-)$ & 9 & & $7(78)$ & & \\
\hline Smear $(+)$ & 27 & & $19(70)$ & & \\
\hline Smear (ND) & 1 & & $1(100)$ & & \\
\hline Patient group 2 & $31(25: 6)$ & $22-77(44.1)$ & $27(87)$ & 87 & \\
\hline Smear (-) & 5 & & $3(60)$ & & \\
\hline Smear $(+)$ & 26 & & $24(92)$ & & \\
\hline Total & $145(68: 77)$ & $19-87(41.6)$ & & & \\
\hline
\end{tabular}

Healthy adults comprised 25 people who visited the health promotion centre of Pusan National University Hospital in whom no evidence of acute or chronic diseases was found by physical examination, chemical tests, haematological tests, serological laboratory tests, gastric endoscopic examination, and radiological tests.

Hospital workers comprised 35 people who had been working in the section dealing with patients with tuberculosis or their specimens for at least one year. No evidence of tuberculosis was found in these people using the periodic acid fast bacilli stain and chest $x$ ray monitoring.

Patients with NTRD comprised 17 inpatients with non-tuberculous respiratory diseases. Tuberculosis was excluded by three consecutive morning sputum stains and cultures, chest $x$ rays, and/or chest computed tomography.

Patient group 1 comprised 37 patients with tuberculosis but no known past history of tuberculosis. Their diagnoses were 33 pulmonary tuberculosis, two pulmonary and intestinal tuberculosis, one tuberculous lymphadenopathy, and one tuberculous meningitis.

Patient group 2 comprised 31 patients with tuberculosis and a previous history of antituberculosis treatment. The mean amount of time between the first and current evidence of tuberculosis was 8.6 years (range, $1-30$ ). The current diagnoses are 30 pulmonary tuberculosis and one pulmonary tuberculosis combined with tuberculous spondylitis.

$\mathrm{M}$, male; F, female; ND, not done.

The hospital workers comprised 35 physicians, nurses, and laboratory technicians who had been working on the tuberculosis wards or mycobacterial laboratories of PNUH or PMC for more than one year. The 17 patients with non-tuberculous respiratory diseases-lung cancer (nine), bacterial pneumonia (four), empyema (two), pleuritis (one), and chronic bronchitis (one)-were randomly selected from the patients who had been hospitalised in the respiratory unit of PNUH. All members of the control groups had no symptoms or signs of tuberculosis at the time of investigation, no previous history of tuberculosis, and no evidence of tuberculosis on chest $x$ rays and AFB stains within six months.

The sera were separated and refrigerated at $4^{\circ} \mathrm{C}$ within three days. The ICT test was performed according to the manufacturer's manual. Briefly, $30 \mu \mathrm{l}$ of serum and three drops of reagent consisting of antihuman IgG coated particles were applied to each side of the membrane, enabling reactions to take place between the membrane fixed antigens and the serum antibodies, followed by antibody-antihuman IgG complex formation. The kit used an internal quality control band, which was seen within 15 minutes of the start of the reaction. The result was considered positive if the control band was observed, and if one or more of the four positive bands appeared. One sample was tested for each individual, and each sample was tested once. The sensitivity and specificity were calculated.

\section{Results}

In patient groups 1 and 2, 27 of $37(73 \%)$ and 27 of $31(87 \%)$ tested positive using ICT, respectively. Of the nine patients with negative AFB stain in patient group 1, seven $(78 \%)$ were ICT positive. Of five patients with negative AFB stain in patient group 2, three $(60 \%)$ showed positive ICT results. Twenty four of 26 $(92 \%)$ and 19 of $27(70 \%)$ of the smear positive patients in patient groups 1 and 2 were ICT positive, respectively. The two patients with extrapulmonary tuberculosis suffering from tuberculous meningitis and lymphadenopathy tested positive using ICT (table 1).

Of the 25 healthy adults, three tested positive with an $88 \%$ specificity. The three were judged to be falsely positive, because sputum AFB staining and culture and chest $x$ rays were negative. Of the 35 hospital workers, two tested positive with a specificity of $94 \%$. These individuals were also considered to be falsely positive because staining and culture with three consecutive morning sputum samples was negative. Of the 17 patients with nontuberculous respiratory disease, one tested positive, with a specificity of $94 \%$.

\section{Discussion}

The role of humoral immunity in the progression of tuberculosis has not been clarified, partly because techniques of purifying antibodies have not been refined. ${ }^{8}$ However, as monoclonal antibody technology progresses some reports claim that the diagnosis of tuberculosis is possible using antigens or antibodies such as LAM, A60 antigen, and TBGL. In addition, there are two methods of diagnosing tuberculosis that use the $38 \mathrm{kDa}$ antigen. One tests for serum $38 \mathrm{kDa}$ antigen using a monoclonal antibody, ${ }^{4}$ and the other uses the epitope of the antigen for detecting serum antibodies. ${ }^{5}$ The sensitivities of the two methods have been reported to be in the range of $68-87 \%$ and $70-92 \%$, respectively. The ICT test is an antibody test kit that reacts with five determinants, including the $38 \mathrm{kDa}$ antigen. It takes $15 \mathrm{~min}$ utes to perform, requires neither special equipment nor special techniques, and costs about $10 \$ \mathrm{US} /$ test, less than the cost of mycobacterial cultures or chest $x$ rays.

In terms of diagnostic sensitivity, it was slightly higher in patient group 2 than in patient group 1. However, in group 2, residual antibody might remain after the primary infection, and could possibly cause a positive result. Therefore, it is reasonable to consider the diagnostic sensitivity as $73 \%$, not the average of the two patient groups. The sensitivity level of $73 \%$ is similar to that of other studies. Ziehl-Neelsen acid fast staining, which is still one of the most widely used tests in screening tuberculosis, shows variable sensitivities in diagnosing tuberculosis, ranging from $30 \%$ to $80 \%$. We suggest that ICT can be used as a supplementary means for diagnosing tuberculosis. However, a considerable number of the smear positive patients $-7.7 \%$ of patient group 2 and $29.6 \%$ of patient group 1-were ICT negative, and these results are probably false negatives. Thus, we believe that ICT cannot replace the chest $x$ ray or AFB stain as a means of quick diagnosis of tuberculosis.

One important consideration when choosing a method for diagnosing tuberculosis is specificity. For a serological antibody to be useful in diagnosing tuberculosis, BCG vaccination or frequent exposure to $M$ tuberculosis should not 
have an effect, because the incidence of tuberculosis in Korea is about $1 \%$ in the general population, and because most children are vaccinated. In our study, tuberculosis in all members of the control groups was excluded by clinical, microbiological, and radiological findings. The individual status of BCG vaccination was unknown, but the nationwide survey of 1995 shows that the vaccination rate was $91.7 \%$ for the population under 30 years of age, and it was estimated as $75 \%$ for the whole age group. Therefore, it seems unlikely that the three controls who were ICT positive were the only ones in this group to have received BCG vaccination, which makes it difficult to say that these positive results were caused by BCG vaccination. In addition, they had no clinical, radiological, or microbiological evidence of tuberculosis, and these results were therefore considered to be false positives. Previous studies found that tests using the $38 \mathrm{kDa}$ antigen had a general specificity of $92-100 \%,{ }^{45} 89$ $91 \%$ in cases of infection by non-tuberculous mycobacteria, ${ }^{59}$ and $89 \%$ in healthy adults exposed to $M$ tuberculosis. ${ }^{10}$ Our study gave similar results-specificity was $88-94 \%$ - and we conclude that the ICT test is highly specific.

It is more difficult to diagnose extrapulmonary tuberculosis than other cases of tuberculosis. As a consequence, it is necessary to adopt molecular biological methods, such as PCR and TMA, for the early diagnosis of extrapulmonary tuberculosis. Wilkins et al have reported that the $38 \mathrm{kDa}$ antibody test used on 73 patients with extrapulmonary tuberculosis (such as tuberculous lymphadenopathy and meningitis) has a sensitivity ranging from $70 \%$ to $81 \%$ (mean, $74 \%$ ). ${ }^{9}$ Although only two patients in our study had extrapulmonary tuberculosis, both tested positive using ICT. In particular, the patient with tuberculous meningitis had been diagnosed as negative by the AFB stain and culture but tested positive using ICT. This indicates that the ICT test could be an important supplementary means for diagnosing extrapulmonary tuberculosis.

1 Park SC, Lee BI, Cho SN, et al. Diagnosis of tuberculous meningitis by detection of immunoglobulin $\mathrm{G}$ antibodies to purified protein derivative and lipoarabinomannan antigen in cerebrospinal fluid. Tuber Lung Dis 1993;74:317-22.

2 Caminero JA, Rodriguez de Castro F, Carrillo T, et al. Value of ELISA using A60 antigen in the serodiagnosis of tuberof ELISA using A60 antigen in the

3 Toyoda T, Osumi M, Aoyagi T, et al. Serodiagnosis of tuberculosis by detection of antituberculous glycolipid antigen (TBGL antigen) antibodies in serum using enzyme-linked mmunosorbent assay: clinical evaluation of anti-TBGL antibodies assay kit. Kekkaku 1996;71:655-61.

4 Bothamley GH, Rudd RM. Clinical evaluation of a serological assay using a monoclonal antibody (TB72) to the $38 \mathrm{kDa}$ antigen of Mycobacterium tuberculosis. Eur Respir f 1994; 7:240-6.

5 Cole RA, Lu HM, Shi YZ, et al. Clinical evaluation of a rapid immunochromatographic assay based on the $38 \mathrm{kDa}$ antigen of Mycobacterium tuberculosis on patients with pulmonary tuberculosis in China. Tuber Lung Dis 1996;77: 363-8.

6 Bothamley G, Batra H, Ramesh V, et al. Serodiagnostic Bothamley G, Batra H, Ramesh V, et al. Serodiagnostic value of the 19 kilodalton antigen of Mycobacterium
tuberculosis in Indian patients. Eur 7 Clin Microbiol Infect Dis 1992;11:912-15.

7 Thierry D, Brisson-Noel A, Vincent-Levy-Frebault V, et al. Characterization of a Mycobacterium tuberculosis insertion sequence, IS and its application in diagnosis. $\mathcal{F}$ Clin Microbiol 1990;28:2668-73.

8 Glatman-Freedman A, Casadevall A. Serum therapy for tuberculosis revisited: reappraisal of the role of antibodymediated immunity against Mycobacterium tuberculosis. Clin Microbiol Rev 1998;11:514-32.

9 Wilkins EG, Ivanyi J. Potential value of serology for diagnosis of extrapulmonary tuberculosis. Lancet 1990;336:6414.

10 Jackett PS, Bothamley GH, Batra HV, et al. Specificity of antibodies to immunodominant mycobacterial antigens in pulmonary tuberculosis. f Clin Microbiol 1988;26:2313-18. 